\title{
Eficácia e seletividade de associações de herbicidas utilizados em pós- emergência na cultura do milho
}

\author{
Efficacy and selectivity of herbicides associations applied in post-emergence in \\ corn crop
}

\author{
Fernando Tadeu de Carvalho ${ }^{1}$, Talita Breda Moretti ${ }^{2}$, Priscila Alves de Souza ${ }^{3}$
}

\begin{abstract}
Resumo - O objetivo do trabalho foi avaliar a seletividade e a eficácia de herbicidas aplicados em pós-emergência para o controle de plantas daninhas em duas cultivares de milho. $\mathrm{O}$ experimento foi desenvolvido entre dezembro/2007 a abril/2008, na FEP-UNESP no município de Selvíria/MS. O experimento foi conduzido em semeadura convencional, utilizando 7 tratamentos e 4 repetições, no esquema de parcelas subdivididas para o fator cultivar: Pioneer 30 S31 e Dow Agroscience 2B710. As aplicações foram realizadas em pós-emergência da cultura e plantas daninhas, aos 7 e 14 dias após a emergência da cultura, utilizando-se um pulverizador pressurizado a $\mathrm{CO}_{2}$ com volume de calda de $200 \mathrm{~L} \mathrm{ha}^{-1}$. Verficou-se que os tratamentos Herbitrin ${ }^{\circledR}+$ Accent $^{\circledR}+$ Joint Oil $^{\circledR}\left(2500 \mathrm{~mL}+25 \mathrm{~g} \mathrm{ha}^{-1}+0,5 \% \mathrm{v} / \mathrm{v}\right) ;$ Herbitrin ${ }^{\circledR}+$ Sanson $40 \mathrm{SC}^{\circledR}$ + Joint Oil ${ }^{\circledR}\left(2500 \mathrm{~mL}+470 \mathrm{~mL} \mathrm{ha}^{-1}+0,5 \% \mathrm{v} / \mathrm{v}\right) ;$ Herbitrin $^{\circledR}+$ Callisto $^{\circledR}+$ Joint Oil $^{\circledR}(2500 \mathrm{~mL}$ $\left.+250 \mathrm{~mL} \mathrm{ha}^{-1}+0,5 \% \mathrm{v} / \mathrm{v}\right)$; e as aplicações seqüenciais de Herbitrin ${ }^{\circledR}+$ Accent $^{\circledR}+$ Joint Oil $^{\circledR}$ $\left(1250 \mathrm{~mL}+21 \mathrm{~g} \mathrm{ha}^{-1}+0,5 \% \mathrm{v} / \mathrm{v}\right) /$ Herbitrin $^{\circledR}+$ Callisto $^{\circledR}+$ Joint Oil $^{\circledR}\left(1250 \mathrm{~mL}+125 \mathrm{~mL} \mathrm{ha}^{-1}+\right.$ $0,5 \% \mathrm{v} / \mathrm{v}) ;$ Herbitrin $^{\circledR}+$ Callisto $^{\circledR}+$ Joint Oil $^{\circledR}\left(1250 \mathrm{~mL}+125 \mathrm{~mL} \mathrm{ha}^{-1}+0,5 \% \mathrm{v} / \mathrm{v}\right) /$ Herbitrin $^{\circledR}+$ Accent $^{\circledR}+$ Joint Oil $^{\circledR}\left(1250 \mathrm{~mL}+21 \mathrm{~g} \mathrm{ha}^{-1}+0,5 \% \mathrm{v} / \mathrm{v}\right)$ e Herbitrin ${ }^{\circledR}+$ Accent $^{\circledR}+$ Callisto $^{\circledR}+$ Joint $\mathrm{Oil}^{\circledR}\left(1250 \mathrm{~mL}+13 \mathrm{~g}+100 \mathrm{~mL} \mathrm{ha}{ }^{-1}+0,5 \% \mathrm{v} / \mathrm{v}\right) /\left(1250 \mathrm{~mL}+13 \mathrm{~g}+100 \mathrm{~mL} \mathrm{ha}^{-1}+0,5 \% \mathrm{v} / \mathrm{v}\right)$ foram altamente seletivos para os híbridos Pioneer 30 S31 e DowAgroceres 2B710, com alta eficácia no controle de Amaranthus deflexus, Ipomoea nil, Commelina benghalensis e Panicum maximum. Conclui-se que os tratamentos podem ser recomendados para o controle de plantas daninhas na cultura do milho.
\end{abstract}

Palavras-chave: Atrazine, nicosulfuron, mesotrione, mistura em tanque, Zea mays.

Abstract - The objective of this work was to evaluate herbicides efficacy and selectivity applied in post-emergence for controlling weeds in two corn cultivars. The experiment was conducted between December/2007 and April/2008, at FEP - UNESP, Selviria County/MS. The experiment was conducted in conventional tillage, by using seven treatments and four replications, in split plot scheme for cultivar factor: Pioneer 30 S31 and Dow Agroscience 2B710. Applications were effected in crops and weeds post-emergence, 7 and 14 days after crop emergence, by using a pressurized sprayer $\left(\mathrm{CO}_{2}\right)$, with spray volume of $200 \mathrm{~L}^{-h^{-1}}$. It was concluded that the treatments: Herbitrin ${ }^{\mathrm{TM}}+$ Accent $^{\mathrm{TM}}+\mathrm{Joint}^{\mathrm{Oil}}{ }^{\mathrm{TM}}\left(2500 \mathrm{~mL}+25 \mathrm{~g}^{- \text {ha }^{-1}}+\right.$ $0,5 \% \mathrm{v} / \mathrm{v}) ;$ Herbitrin $^{\mathrm{TM}}+$ Sanson $40 \mathrm{SC}^{\mathrm{TM}}+\mathrm{Joint} \mathrm{Oil}^{\mathrm{TM}}\left(2500 \mathrm{~mL}+470 \mathrm{~mL} \mathrm{ha}^{-1}+0,5 \% \mathrm{v} / \mathrm{v}\right)$; Herbitrin $^{\mathrm{TM}}+$ Callisto $^{\mathrm{TM}}+$ Joint Oil $^{\mathrm{TM}}\left(2500 \mathrm{~mL}+250 \mathrm{~mL} \mathrm{ha}{ }^{-1}+0,5 \% \mathrm{v} / \mathrm{v}\right)$; and sequential applications of Herbitrin ${ }^{\mathrm{TM}}+$ Accent $\left.^{\mathrm{TM}}+\operatorname{Joint~Oil~}^{\mathrm{TM}}\left(1250 \mathrm{~mL}+21 \mathrm{~g} \mathrm{ha}^{-1}+0,5 \% \mathrm{v} / \mathrm{v}\right)\right) /$

${ }^{1}$ Professor Dr., DBZ-FEIS-UNESP, Ilha Solteira/SP, Brasil. E-mail: ftadeu@ bio.feis.unesp.br;

${ }^{2}$ Pós-graduanda de Agronomia, FEIS-UNESP, Ilha Solteira/SP;

${ }^{3}$ Aluna do curso de Agronomia Universidade Castelo Branco - UNICASTELO, Fernandópolis/SP.

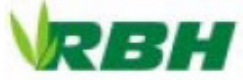


Herbitrin $^{\mathrm{TM}}+$ Callisto $^{\mathrm{TM}}+$ Joint Oil $^{\mathrm{TM}}\left(1250 \mathrm{~mL}+125 \mathrm{~mL} \mathrm{ha}^{-1}+0,5 \% \mathrm{v} / \mathrm{v}\right) ;$ Herbitrin $^{\mathrm{TM}}+$ Callisto $^{\mathrm{TM}}+$ Joint Oil $^{\mathrm{TM}}\left(1250 \mathrm{~mL}+125 \mathrm{~mL} \mathrm{ha}^{-1}+0,5 \% \mathrm{v} / \mathrm{v}\right) /$ Herbitrin $^{\mathrm{TM}}+$ Accent $^{\mathrm{TM}}+$ Joint $\mathrm{Oil}^{\mathrm{TM}}\left(1250 \mathrm{~mL}+21 \mathrm{~g} \mathrm{ha}^{-1}+0,5 \% \mathrm{v} / \mathrm{v}\right)$ and Herbitrin ${ }^{\mathrm{TM}}+$ Accent $^{\mathrm{TM}}+$ Callisto $^{\mathrm{TM}}+$ Joint Oil $^{\mathrm{TM}}$ $\left(1250 \mathrm{~mL}+13 \mathrm{~g}+100 \mathrm{~mL} \mathrm{ha}^{-1}+0,5 \% \mathrm{v} / \mathrm{v}\right) /\left(1250 \mathrm{~mL}+13 \mathrm{~g}+100 \mathrm{~mL} \mathrm{ha}^{-1}+0,5 \% \mathrm{v} / \mathrm{v}\right)$ were highly selective to Pioneer 30 S31 and Dow Agroceres 2B710 cultivars, with expressive efficacy in controlling Amaranthus deflexus, Ipomoea nil, Commelina benghalensis e Panicum maximum weeds. It was also observed that these treatments may be recommended for controlling weed in corn crop.

Key-words: Atrazine, nicosulfuron, mesotrione, association, postemergence.

\section{Introdução}

A cultura do milho está entre as mais importantes do Brasil com uma área plantada em torno de 14 milhões de hectares e produtividade média de $3.670 \mathrm{~kg} \mathrm{ha}^{-1}$ (IBGE, 2009). As condições edafoclimáticas destacam o Brasil como país de grande potencial para a cultura do milho, entretanto, o clima tropical é também muito propício à ocorrência de grande quantidade de plantas daninhas, que interferem no desenvolvimento e na produtividade da cultura. Os efeitos negativos detectados no crescimento e na produtividade da cultura devido à presença das plantas daninhas foram observados por vários autores (Carvalho \& Galli, 1993; Velini et al., 1993; Silva et al., 1993).

Dentre os fatores que provocam interferência destaca-se o período em que a população de plantas daninhas compete pelos recursos do ambiente com a cultura, no qual se torna necessário o uso de medidas de controle para reduzir os efeitos negativos dessa interferência (Silva et al., 2002). Dentre estas, o controle químico tem se destacado, pela eficiência no controle das plantas daninhas, rapidez na operação e economia nos custos, quando comparados com outros métodos. Todavia, a eficácia dos herbicidas é variável e dependente das condições ambientais, da época de aplicação e da espécie daninha a ser controlada (Merotto Jr. et al., 1997).
Existem diversos métodos para se controlar as plantas daninhas na agricultura. Os métodos mais utilizados são os mecânicos e o controle químico. No milho, a utilização de herbicidas tem se mostrado uma ótima opção em função do grande rendimento operacional que se consegue, e devido às desvantagens dos métodos mecânicos, pouco eficientes quando utilizados em períodos chuvosos e com alta umidade do solo.

Atualmente, a maioria dos herbicidas registrados para cultura do milho são de aplicação em pré-emergência. $O$ estudo da eficácia de novas formulações de herbicidas, principalmente para aplicação em pósemergência, é muito importante no sentido de se encontrar opções para a agricultura com a vantagem se aproveitar o período em que as ervas são mais sensíveis. O nicosulfuron é uma das poucas opções de herbicida pós-emergente seletivo para a cultura do milho, com eficácia em plantas daninhas do grupo das monocotiledôneas e dicotiledôneas (Rodrigues \& Almeida, 2005).

Desta forma o presente trabalho foi desenvolvido com objetivo de avaliar a seletividade e a eficácia de herbicidas aplicados em pós-emergência para o controle de plantas daninhas em dois cultivares de milho.

\section{Material e Métodos}


O experimento foi desenvolvido no período de dezembro/2007 a abril/2008, em área irrigada da Fazenda de Ensino e Pesquisa (FEP) da FEIS-UNESP, localizada no município de Selvíria, MS, enquadrada em região de cerrado, no Mato Grosso do Sul. O solo da área experimental é classificado como Latossolo Vermelho-Escuro textura argilosa, com $44 \%$ de argila, $8 \%$ de silte e $48 \%$ de areia. As coordenadas da área experimental são $20^{\circ} 20^{\prime} 47,9^{\prime \prime}$ de latitude sul e $51^{\circ} 24^{\prime} 11,8^{\prime \prime}$ de longitude oeste, com 342 metros de altitude.

$\mathrm{O}$ experimento foi implantado em área de cultivo convencional preparado com uma aração e duas gradagens niveladoras, sendo a última gradagem, imediatamente antes da semeadura, de modo a eliminar as plantas daninhas que já haviam germinado. A semeadura foi realizada em 11/12/2007, utilizando-se a máquina semeadora pneumática Jumil modelo 2600. Os híbridos de milho Pioneer 30 S31 e DowAgroscience 2B710 foram semeados com espaçamento de $1,0 \mathrm{~m}$ entrelinhas e 5,4 sementes por metro. A emergência ocorreu cinco dias após a semeadura, em 16/12/2007.

A adubação foi realizada concomitantemente à semeadura, utilizando-se a fórmula comercial de NPK 8-28-16 + 0,3\% de Zinco à base de $240 \mathrm{~kg} \mathrm{ha}^{-1}$, e adubação de cobertura, aos 30 dias após a semeadura, com $220 \mathrm{~kg} \mathrm{ha}^{-1}$ do formulado NPK 20-0-20. A aplicação do inseticida fisiológico lefenurom (Match EC $^{\circledR} 300 \mathrm{~mL} \mathrm{ha}^{-1}$ ) para o controle de lagartas-do-cartucho (Spodoptera frugiperda) foi realizado com pulverizador de barra tratorizado, utilizando volume de calda de 200 $\mathrm{L} \mathrm{ha}^{-1}$.

$\mathrm{O}$ delineamento experimental adotado foi o de blocos ao acaso com sete tratamentos e quatro repetições, no esquema de parcelas subdivididas para o fator cultivar: Pioneer 30 S31 e DowAgroscience 2B710. Cada parcela constou de três linhas de cada cultivar com $5 \mathrm{~m}$ de comprimento e $6 \mathrm{~m}$ de largura, totalizando $30 \mathrm{~m}^{2}$. Os tratamentos com suas respectivas doses e época de aplicação estão representados na Tabela 1.

As aplicações dos herbicidas foram realizadas com pulverizador costal pressurizado a $\mathrm{CO}_{2}$, com pressão constante de $45 \mathrm{lb} \mathrm{pol}^{-2}$, provido de tanque com capacidade de dois litros (garrafas descartáveis), equipado com barra de quatro pontas do tipo leque Teejet XR 110.03, espaçadas de meio metro, que proporcionou um volume de calda aplicado de $200 \mathrm{~L} \mathrm{ha}^{-1}$. As aplicações foram realizadas em pós-emergência da cultura e plantas daninhas, aos 7 dias após a emergência (DAE) (23/12/2008), e nos tratamentos (5, 6 e 7) com aplicação sequencial aos 14 DAE (30/12/2008). No momento das aplicações o solo encontravase com umidade; a temperatura média do ambiente foi de $30^{\circ} \mathrm{C}$, a UR do ar superior a $60 \%$ e a velocidade do vento inferior a $2 \mathrm{~km} \mathrm{~h}^{-}$ 1.

As avaliações de seletividade foram realizadas até o desaparecimento dos sintomas de fitotoxicidade aos 7 e 15 dias após a aplicação (DAA). Para se determinar o efeito fitotóxico dos herbicidas utilizou-se uma escala visual em porcentagem de injúria, onde $0 \%$ representou nenhum sintoma e $100 \%$ a morte total da cultura. As avaliações de controle de plantas daninhas foram realizadas até o fechamento da cultura, aos 7, 15, 30 e 45 DAA e na pré-colheita. Utilizou-se uma escala visual de notas, onde $0 \%$ representou nenhum controle e $100 \%$ o controle total das plantas daninhas. Considerou-se como eficiente o controle igual ou superior a $80 \%$, conforme metodologia proposta por SBCPD (1995). A avaliação de produtividade foi realizada aos 125 dias após a semeadura (13/04/2008), coletando-se os grãos da área útil $\left(2 \mathrm{~m}^{2}\right.$ centrais de cada parcela). Os dados coletados foram submetidos à análise de variância pelo teste $\mathrm{F}$ e analisados estatisticamente através do teste de Tukey a $5 \%$ de probabilidade. 
Tabela 1. Tratamentos com doses e época de aplicação, utilizados no experimento.

\begin{tabular}{|c|c|c|c|}
\hline \multirow{2}{*}{ Tratamentos } & \multicolumn{2}{|c|}{ Dose dos herbicidas } & \multirow{2}{*}{$\begin{array}{l}\text { Época de } \\
\text { Aplicação }\end{array}$} \\
\hline & p.c. g ou mL ha ${ }^{-1}$ & i.a. $\left(\mathrm{g} \mathrm{ha}^{-1}\right)$ & \\
\hline 1- Testemunha sem controle (no mato) & -- & -- & -- \\
\hline 2- Herbitrin $^{\circledR}+$ Accent $^{\circledR}+$ Joint Oil $^{\circledR}$ & $2500+25$ & $1250+18,75$ & $7 \mathrm{DAE}$ \\
\hline 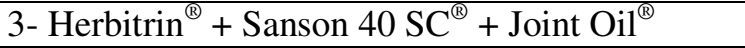 & $2500+470$ & $1250+18,80$ & $7 \mathrm{DAE}$ \\
\hline 4- Herbitrin ${ }^{\circledR}+$ Callisto $^{\circledR}$ & $2500+250$ & $1250+138,80$ & $7 \mathrm{DAE}$ \\
\hline $\begin{array}{l}\text { 5- Herbitrin }{ }^{\circledR}+\text { Accent }^{\circledR}+\text { Joint Oil }^{\circledR} / \\
\text { Herbitrin }^{\circledR}+\text { Callisto }^{\circledR}+\text { Joint Oil }^{\circledR}\end{array}$ & $\begin{array}{c}1250+21 \\
1250+125\end{array}$ & $\begin{array}{l}625+15,75 / \\
625+60\end{array}$ & $\begin{array}{l}7 \mathrm{DAE} / \\
14 \mathrm{DAE}\end{array}$ \\
\hline $\begin{array}{l}\left.\text { 6- Herbitrin }{ }^{\circledR}+\text { Callisto }^{(}\right)+ \text {Joint Oil }{ }^{\circledR} / \\
\text { Herbitrin }^{\circledR}+\text { Accent }^{\circledR}+\text { Joint Oil }^{\circledR}\end{array}$ & $\begin{array}{c}1250+125 \\
1250+21\end{array}$ & $\begin{array}{c}625+60 / \\
625+15,75\end{array}$ & $\begin{array}{l}7 \mathrm{DAE} / \\
14 \mathrm{DAE}\end{array}$ \\
\hline $\begin{array}{c}\text { 7- Herbitrin }{ }^{\circledR}+\text { Accent }^{\circledR}+\text { Callisto }^{(}{ }^{\circledR}+\text { Joint Oil }^{\circledR} / \\
\text { Herbitrin }^{\circledR}+\text { Accent }^{\circledR}+\text { Callisto }^{\circledR}+\text { Joint Oil }^{\circledR}\end{array}$ & $\begin{array}{l}1250+13+100 \\
1250+13+100\end{array}$ & $\begin{array}{l}625+9,75+48 / \\
625+9,75+48\end{array}$ & $\begin{array}{l}7 \mathrm{DAE} / \\
14 \mathrm{DAE}\end{array}$ \\
\hline
\end{tabular}

Obs.: i.a. = ingrediente ativo; Joint $\mathrm{Oil}^{\circledR}=$ óleo mineral utilizado na dose de 0,5\% v/v; DAE = Dias Após a Emergência; Tratamentos 5, 6 e 7: $1^{\underline{a}}$ aplicação realizada aos 7 DAE (milho com 4 folhas) e aplicação seqüencial aos 14 DAE (milho com 6 folhas).

\section{Resultados e Discussão}

A densidade de plantas daninhas na área experimental, no momento da aplicação dos tratamentos, foi de 22,5 plantas $\mathrm{m}^{-2}$ de Amaranthus deflexus (caruru AMADE); 10,0 plantas $\mathrm{m}^{-2}$ de Ipomoea nil (corda-de-viola IPONI), com 2 a 3 pares de folhas; 11,5 plantas $\mathrm{m}^{-2}$ de Commelina benghalensis (trapoeraba COMBE) com 2 a 3 folhas e 10,0 plantas $\mathrm{m}^{-2}$ de Panicum maximum (capim-colonião PANMA) com 1 perfilho. Os dados de porcentagem de controle das plantas daninhas aos 45 DAA estão apresentados na Tabela 2.

Tabela 2. Eficácia no controle das plantas daninhas aos 45 DAA. FEP, Selvíria/MS, 2008.

\begin{tabular}{|c|c|c|c|c|c|}
\hline \multirow[b]{2}{*}{ Tratamentos } & \multirow{2}{*}{$\begin{array}{l}\text { Dose } \\
\text { p.c.mL ou } \mathrm{g} \mathrm{ha}^{-1}\end{array}$} & \multicolumn{4}{|c|}{$\%$ Controle aos 45 DAA } \\
\hline & & AMADE & IPONI & COMBE & PANMA \\
\hline 1- Testemunha sem controle (no mato) & -- & 0,0 & 0,0 & 0,0 & 0,0 \\
\hline 2- Herbitrin ${ }^{\circledR}+$ Accent $^{\circledR}+$ Joint Oil $^{\circledR}$ & $2500+25$ & 100,0 & 98,5 & 98,0 & 99,3 \\
\hline 3- Herbitrin ${ }^{\circledR}+$ Sanson 40 SC $^{\circledR}+$ Joint Oil $^{\circledR}$ & $2500+470$ & 100,0 & 98,5 & 97,5 & 99,8 \\
\hline 4- Herbitrin ${ }^{\circledR}+$ Callisto $^{\circledR}$ & $2500+250$ & 100,0 & 99,5 & 99,3 & 99,5 \\
\hline $\begin{aligned} & \text { 5- Herbitrin }{ }^{\circledR} \\
& \text { Herbitrin }^{\circledR}+ \text { Accent }^{\circledR}+\text { Callisto }^{\circledR}+\text { Joint Oint Oil }^{\circledR} \\
&\end{aligned}$ & $\begin{array}{c}1250+21 \\
1250+125\end{array}$ & 100,0 & 100,0 & 99,5 & 100,0 \\
\hline $\begin{array}{l}\text { 6- Herbitrin }{ }^{\circledR}+\text { Callisto }^{\circledR}+\text { Joint Oil }^{\circledR} / \\
\text { Herbitrin }^{\circledR}+\text { Accent }^{\circledR}+\text { Joint Oil }^{\circledR}\end{array}$ & $\begin{array}{c}1250+125 \\
1250+21\end{array}$ & 100,0 & 100,0 & 99,5 & 99,8 \\
\hline $\begin{array}{l}\text { 7- Herbitrin }{ }^{\circledR}+\text { Accent }^{\circledR}+\text { Callisto }^{\circledR}+\text { Joint Oil }^{\circledR} / \\
\text { Herbitrin }^{\circledR}+\text { Accent }^{\circledR}+\text { Callisto }^{\circledR}+\text { Joint Oil }^{\circledR}\end{array}$ & $\begin{array}{l}1250+13+100 \\
1250+13+100\end{array}$ & 100,0 & 100,0 & 99,5 & 99,8 \\
\hline
\end{tabular}

Obs.: tratamentos 2, 3 e 4 aplicados aos 7 DAE e tratamentos 5, 6 e 7 aplicados em seqüencial 7 DAE/14 DAE.

Observou-se pelos dados de controle das plantas daninhas que os tratamentos foram altamente eficientes, proporcionando, em média, níveis de controle superiores a 97,5\% aos 45 DAA. Para a espécie A. deflexus (AMADE) o controle proporcionado pelos tratamentos foi total $(100,0 \%)$, apesar de ter ocorrido uma redução natural da infestação da 
planta daninha a partir dos 45 dias após a emergência do milho. Para as espécies $I$. nil (IPONI), $C$. benghalensis (COMBE) e $P$. maximum (PANMA), os tratamentos foram altamente eficientes, com níveis de controle superiores a 98,5\%, 97,5\% e 99,3\%, respectivamente. Entre as espécies de plantas daninhas estudadas apenas o caruru (A. deflexus) é sensível a atrazina, segundo Lorenzi et al. (2006). Portanto as misturas em tanque com nicosulfuron e/ou mesotrione foram importantes para o controle eficaz das demais espécies: I. nil, C. benghalensis e $P$. maximum.

Tabela 3. Teste de Tukey para os dados de produtividade do milho dentro do fator manejo de plantas daninhas. FEP, Selvíria/MS, 2008.

\begin{tabular}{|c|c|c|c|}
\hline \multirow[b]{2}{*}{ Tratamentos } & \multirow[b]{2}{*}{$\begin{array}{l}\text { Dose } \\
\text { p.c.mL ou g ha }\end{array}$} & \multicolumn{2}{|c|}{ Produtividade $\left(\mathrm{kg} \mathrm{ha}^{-1}\right)$} \\
\hline & & $\begin{array}{c}\text { Cultivar: } \\
\text { Pioneer 30S31 }\end{array}$ & $\begin{array}{c}\text { Cultivar: } \\
\text { DowAg. } \\
\text { 2B710 }\end{array}$ \\
\hline 1- Testemunha sem controle (no mato) & -- & $7011,8 \mathrm{~b}$ & $6206,3 \mathrm{~b}$ \\
\hline 2- Herbitrin ${ }^{\circledR}+$ Accent $^{\circledR}+$ Joint Oil $^{\circledR}$ & $2500+25$ & 8677,8 a & 7308,9 a \\
\hline 3- Herbitrin ${ }^{\circledR}+$ Sanson $40 \mathrm{SC}^{\circledR}+$ Joint Oil $^{\circledR}$ & $2500+470$ & 8651,7 a & 7281,5 a \\
\hline 4- Herbitrin ${ }^{\circledR}+$ Callisto $^{\circledR}$ & $2500+250$ & 8492,8 a & 7126,6 a \\
\hline $\begin{array}{l}\text { 5- Herbitrin }{ }^{\circledR}+\text { Accent }^{\circledR}+\text { Joint Oil }^{{ }^{\circledR}} / \\
\text { Herbitrin }^{\circledR}+\text { Callisto }^{\circledR}+\text { Joint Oil }^{\circledR}\end{array}$ & $\begin{array}{c}1250+21 \\
1250+125\end{array}$ & 8733,7 a & 7263,1 a \\
\hline $\begin{array}{l}\text { 6- Herbitrin }{ }^{\circledR}+\text { Callisto }^{\circledR}+\text { Joint Oil }{ }^{\circledR} / \\
\text { Herbitrin }^{\circledR}+\text { Accent }^{\circledR}+\text { Joint Oil }^{\circledR}\end{array}$ & $\begin{array}{c}1250+125 \\
1250+21\end{array}$ & 8557,2 a & 7361,2 a \\
\hline $\begin{array}{c}\text { 7- Herbitrin }{ }^{\circledR}+\text { Accent }^{\circledR}+\text { Callisto }^{\circledR}+\text { Joint Oil }^{\circledR} / \\
\text { Herbitrin } \\
{ }^{\circledR}+\text { Accent }^{\circledR}+\text { Callisto }^{\circledR}+\text { Joint Oil }^{\circledR}\end{array}$ & $\begin{array}{l}1250+13+100 \\
1250+13+100 \\
\end{array}$ & 8858,1 a & 7246,2 a \\
\hline \multicolumn{2}{|l|}{ Média } & 8426,17 & 7113,41 \\
\hline \multicolumn{2}{|l|}{ Causas de Variação } & \multicolumn{2}{|c|}{ Teste F } \\
\hline \multicolumn{2}{|l|}{ fator A = TRATAMENTOS (parcela) } & \multicolumn{2}{|c|}{$7,31 * *$} \\
\hline \multicolumn{2}{|l|}{ fator B = CULTIVAR (sub-parcela) } & \multicolumn{2}{|c|}{$79,25 * *$} \\
\hline \multicolumn{2}{|l|}{ fator $\mathrm{AxB}$} & \multicolumn{2}{|c|}{$0,43^{\mathrm{NS}}$} \\
\hline \multicolumn{2}{|l|}{ Coeficiente de variação } & \multicolumn{2}{|c|}{$7,10 \%$} \\
\hline
\end{tabular}

Obs: tratamentos 2, 3 e 4 aplicados aos 7 DAE e tratamentos 5, 6 e 7 aplicados em seqüencial aos 7 DAE/14 DAE.

- Médias seguidas de letras iguais nas colunas não diferem entre si pelo teste de Tukey (5\%).** resultado significativo ao nível de $1 \%$ de probabilidade.
$\mathrm{Na}$ avaliação realizada na pré-colheita da cultura do milho observou-se que os tratamentos mantiveram o controle residual sobre $I$. nil e $P$. maximum. Para a $C$. benghalensis, o controle residual foi ineficaz (< $80,0 \%$ ) em todos os tratamentos, mas por se tratar do início da reinfestação da planta daninha, não houve prejuízo na produtividade, assim como não foram constatados limitações e/ou dificuldades na operação de colheita da cultura. 
Nas avaliações de seletividade, observou-se que os tratamentos herbicidas foram altamente seletivos para os dois híbridos de milho estudados, sendo apenas constatados baixos níveis de fitotoxicidade $(<2,5 \%)$. Aos 30 DAA, não foi observado sintomas nas plantas de milho. López-Ovejero (2003) e Foloni (2002) também observaram alta seletividade do milho ao nicosulfuron e atrazina e ao mesotrione, respectivamente. Os dados médios de produtividade da cultura estão apresentados na Tabela 3, onde os valores de F apontam que houve significância dentro dos fatores, ou seja, que ocorreu diferença significativa entre os cultivares e entre os tratamentos utilizados no manejo de plantas daninhas.

No que diz respeito aos dados de produtividade do milho, dentro do fator manejo de plantas daninhas (Tabela 3), observa-se que a ocorrência das plantas daninhas prejudicou significativamente o rendimento da cultura no tratamento testemunha na ausência de controle, para ambos os cultivares. Os dados de produtividade confirmam os efeitos prejudiciais da competição entre cultura e plantas daninhas, verificados por outros autores (Carvalho \& Galli, 1993; Velini et al., 1993 e Silva et al., 1993).

\section{Conclusões}

Considerando-se os resultados obtidos concluiu-se que os tratamentos Herbitrin ${ }^{\circledR}+$ Accent $^{\circledR}+$ Joint Oil $^{\circledR}\left(2500 \mathrm{~mL}+25 \mathrm{~g} \mathrm{ha}^{-1}+\right.$ $0,5 \% \mathrm{v} / \mathrm{v}) ;$ Herbitrin $^{\circledR}+$ Sanson $40 \mathrm{SC}^{\circledR}+$ Joint $\mathrm{Oil}^{\circledR}\left(2500 \mathrm{~mL}+470 \mathrm{~mL} \mathrm{ha}{ }^{-1}+0,5 \% \mathrm{v} / \mathrm{v}\right)$; Herbitrin $^{\circledR}+$ Callisto $^{\circledR}+$ Joint Oil $^{\circledR}(2500 \mathrm{~mL}$ $\left.+250 \mathrm{~mL} \mathrm{ha}^{-1}+0,5 \% \mathrm{v} / \mathrm{v}\right)$; e as aplicações sequenciais de Herbitrin ${ }^{\circledR}+$ Accent $^{\circledR}+$ Joint $\mathrm{Oil}^{\circledR}\left(1250 \mathrm{~mL}+21 \mathrm{~g} \mathrm{ha}^{-1}+0,5 \% \mathrm{v} / \mathrm{v}\right) /$ Herbitrin $^{\circledR}+$ Callisto $^{\circledR}+$ Joint Oil $^{\circledR}(1250 \mathrm{~mL}$ $\left.+125 \mathrm{~mL} \mathrm{ha}^{-1}+0,5 \% \mathrm{v} / \mathrm{v}\right)$; Herbitrin ${ }^{\circledR}+$ Callisto ${ }^{\circledR}+$ Joint Oil $^{\circledR}\left(1250 \mathrm{~mL}+125 \mathrm{~mL} \mathrm{ha}^{-1}\right.$ $+0,5 \% \mathrm{v} / \mathrm{v}) /$ Herbitrin $^{\circledR}+$ Accent $^{\circledR}+$ Joint Oil $^{\circledR}$ $\left(1250 \mathrm{~mL}+21 \mathrm{~g} \mathrm{ha}^{-1}+0,5 \% \mathrm{v} / \mathrm{v}\right)$ e Herbitrin ${ }^{\circledR}$ + Accent $^{\circledR}+$ Callisto $^{\circledR}+$ Joint Oil $^{\circledR}(1250 \mathrm{~mL}$ $\left.+13 \mathrm{~g}+100 \mathrm{~mL} \mathrm{ha}{ }^{-1}+0,5 \% \mathrm{v} / \mathrm{v}\right) /(1250 \mathrm{~mL}+$ $13 \mathrm{~g}+100 \mathrm{~mL} \mathrm{ha}{ }^{-1}+0,5 \% \mathrm{v} / \mathrm{v}$ ) foram altamente seletivos para os híbridos Pioneer 30 S31 e DowAgroceres 2B710.

Todos os tratamentos apresentaram alta eficácia no controle de Amaranthus deflexus, Ipomoea nil, Commelina benghalensis e Panicum maximum, podendo serem recomendados para o controle de plantas daninhas na cultura do milho.

\section{Referências}

CARVALHO, F.T.; GALLI, A.J.B. Nova formulação de alachlor na seletividade e no controle de plantas daninhas na cultura do milho. In: CONGRESSO BRASILEIRO DE HERBICIDAS E PLANTAS DANINHAS, 19, 1993 Londrina, PR. Anais... Londrina: SBHED, 1993. p. 131.

FOLONI, L.L. Callisto (mesotrione) - um novo herbicida pós-emergente para a cultura do milho (Zea mays L.). In: CONGRESSO BRASILEIRO DA CIÊNCIA DAS PLANTAS DANINHAS, 23, 2002, Gramado, RS, Anais... Londrina, PR: SBCPD, 2002. p. 308.

IBGE - Instituto Brasileiro de Geografia e Estatística. Levantamento Sistemático da Produção Agrícola - Abril / Maio 2009 http://www.ibge.gov.br/home/estatistica /indicadores/agropecuária/lspa/lspa _200905_3.shtm.

LÓPEZ-OVEJERO, R.F. et al. Seletividade de herbicidas para a cultura de milho (Zea mays) aplicados em diferentes estádios fenológicos da cultura. Planta Daninha, v.21, n.3, p.413419, 2003. 
LORENZI, H. et al. Manual de identificação e controle de plantas daninhas: plantio direto e convencional. $6^{\mathrm{a}}$ Ed., Nova Odessa, SP: Plantarum, 2006. 339 p.

MEROTTO JR., A. et al. Aumento da população de plantas e uso de herbicidas no controle de plantas daninhas em milho. Planta Daninha, v.15, n.2, p.141-151, 1997.

RODRIGUES, B.N.; ALMEIDA, F.S. Guia de herbicidas. $5^{\mathrm{a}}$ Ed., Londrina: Edição dos Autores, 2005. 592 p.

SOCIEDADE BRASILEIRA DA CIÊNCIA DAS PLANTAS DANINHAS - SBCPD. Procedimentos para instalação, avaliação e análise de experimentos com herbicidas. Londrina: SBCPD, 42 p., 1995.

SILVA, A. A. et al. Biologia e controle de plantas daninhas. Viçosa: DFT/UFV, 2002. CD-ROM.

SILVA, M.S.J. et al. Estudo do herbicida nicosulfuron no controle de plantas daninhas na cultura do milho (Zea mays L.). In: CONGRESSO BRASILEIRO DE HERBICIDAS E PLANTAS DANINHAS, 19, 1993, Londrina, PR. Anais... Londrina: SBHED, 1993. p. 148-149.

VELINI, E. et al. Eficiência de herbicidas pré e pós emergentes iniciais recomendados para a cultura do milho, no controle de plantas daninhas e avaliação de efeitos dos mesmos sobre a produtividade da cultura. In: CONGRESSO BRASILEIRO DE HERBICIDAS E PLANTAS DANINHAS, 19, 1993, Londrina, PR. Anais... Londrina: SBHED, 1993. p. 141-142. 